\title{
Therapeutic Strategies of Kidney Transplant Ischemia Reperfusion Injury: Insight From Mouse Models
}

\author{
Longhui Qiu ${ }^{1}$ and Zheng Jenny Zhang*1,2 \\ ${ }^{1}$ Comprehensive Transplant Center, USA \\ ${ }^{2}$ Department of Surgery, USA
}

*Corresponding author: Zheng Jenny Zhang, Department of Surgery, Comprehensive Transplant Center, Feinberg School of Medicine, USA

\begin{abstract}
ARTICLE INFO
Received: 业 February 07, 2019

Published: 幽 February 20, 2019

Citation: Longhui Q, Zheng Jenny Z. Therapeutic Strategies of Kidney Transplant Ischemia Reperfusion Injury: Insight From Mouse Models. Biomed J Sci \& Tech Res 14(5)-2019. BJSTR. MS.ID.002617.
\end{abstract}

Keywords: Therapies; Kidney; Transplantation; Ischemia Reperfusion Injury; Mouse Models; Cold Ischemic Time; Complement; Regeneration

\section{ABSTRACT}

Ischemia/reperfusion injury (IRI) is inherent to all transplanted organs and is adversely associated with early renal graft function and graft longevity. Despite the progress in immunosuppressive regimens and perioperative care, no FDA-approved treatment for kidney transplant IRI is available to date. In recent years, by utilizing the modified and clinically-relevant mouse models of kidney transplantation (KT) in which extended IRI is induced by the prolonged warm or cold ischemic time, studies have identified several potential therapeutic approaches for KT IRI, including the hormone supplement, promoting tubular repair and regeneration, and targeting complement system, inflammation, and necroptosis. This review describes some of the lessons learned from mouse models of KT with regard to factors that influence the severity of transplant IRI and the potential therapeutic targets.

Abbreviations: ESRD: End Stage Renal Disease; KT: Kidney Transplantation; IRI: Ischemia-Reperfusion Injury; ROS: Reactive Oxygen Species; ATN: Acute Tubular Necrosis; DGF: Delayed Graft Function; CIT: Cold Ischemic Time; WIT: Warm Ischemic Time; $\beta$-hCG: $\beta$-Human Chorionic Gonadotropin; AKI: Acute Kidney Injury; IL-6: interleukin-6; TNF- $\alpha$ : Tumor Necrosis Factor- $\alpha$; TLRs: Toll like Receptors; RIPK: Receptor Interacting Protein Kinase; NO-HDL-NPs: Nitric Oxide Delivering High Density Lipoprotein Like Nanoparticles

\section{Introduction}

End-stage renal disease (ESRD) is a global public health problem with generally poor outcomes and high costs. According to the latest U.S. Renal Data System Annual Data Report (2018), more than 720,000 Americans are being treated for ESRD. Kidney transplantation (KT) has become the optimal treatment for ESRD. However, all transplanted kidneys inevitably experience ischemia/ reperfusion injury (IRI) - the restriction of blood flow and the restoration of blood supply during transplant process trigger a cascade of noxious events, including the microvascular alteration, reactive oxygen species (ROS) responses, cytokine and chemokine release, and leukocyte activation, resulting in sterile inflammation and acute tubular necrosis (ATN) in renal grafts [1,2]. Severe transplant IRI frequently translates into delayed graft function (DGF), a common complication associated with a high morbidity and mortality post-KT and allograft rejection [1,3-5].
With a growing gap between kidney demand and supply, the quality of the donor kidney pool continues to deteriorate as the increasing donor age and associated comorbidities accumulate $[6,7]$. Expanded criteria donor kidneys of perceived lower quality are particularly susceptible to IRI, which compromises graft outcomes [7]. Therefore, seeking strategies to understand the pathophysiology of KT IRI and to develop effective treatments is highly desirable for transplant recipients [8]. In recent years, our laboratory and others have been attempting to adapt the mouse KT into a more clinically feasible model to investigate the mechanisms and new therapeutic approaches of transplant IRI. In this minireview, we discuss some of the lessons learned from mouse models of KT with regard to factors that influence the severity of transplant IRI and the potential therapeutic targets. 
Clinically Relevant Mouse Models of Kidney Transplantation With Extended IRI

Mouse models of vascularized kidney transplantation have been widely used to dissect mechanism of IRI and transplant rejection. Similarly to the clinic, prolonged cold ischemic time (CIT) induce extended IRI in mouse renal grafts, thus introducing a clinically-relevant model for transplant IRI studies (Table 1) [9-17]. While the warm ischemic time (WIT) in most studies was limited to $30 \mathrm{mins}$, the CIT ranged from 20 mins to $8 \mathrm{hrs}$. In some studies, both of the native kidneys of recipient mice were removed during the transplant surgery, while in other studies one of the recipient's native kidneys was kept in situ until day 4-5 post-transplant. The advantage of removing both native kidneys right away is that the immediate renal graft function will be accessible; however, it may also cause more mortalities during the early post-transplant phases due to the intensive IRI. Concerning the donor and recipient strains, both syngeneic and allogeneic mouse models have been employed for testing IRI treatments. However, since the contributions remain unclear with respect to the allogeneic immune responses versus donor/recipient genetics in graft IRI, the influence of mouse genetic background should be also taken into consideration when designing studies.

Table 1: Potential therapies or targets of kidney transplant IRI identified by utilizing the modified mouse models of KT with extended IRI.

\begin{tabular}{|c|c|c|c|c|}
\hline Author & Year & Mouse KT Models & Therapies/Targets & Reference \\
\hline \multicolumn{5}{|c|}{ Hormone } \\
\hline David et al. & 2016 & Syngeneic; 8hr CIT; 30 min WIT * & Estrogen & [9] \\
\hline Gueler et al. & 2015 & Allogeneic; $1 \mathrm{hr}$ CIT; $30 \mathrm{~min}$ WIT* & EA-230 & {$[10]$} \\
\hline \multicolumn{5}{|c|}{ Targeting Complement System } \\
\hline Zhang et al. & 2016 & Syngeneic; $4 \mathrm{hr} \mathrm{CIT}^{\#} \dagger$ & $\begin{array}{c}\text { Complement } 3 \text {, RelB, and } \\
\text { Fas }\end{array}$ & [11] \\
\hline Casuraghi et al. & 2017 & Allogeneic; 20min CIT; 20 min WIT" & Complement factor $\mathrm{b}$ & {$[12]$} \\
\hline \multicolumn{5}{|c|}{ Tubular Repair and Regeneration } \\
\hline Zhang et al. & 2016 & Syngeneic; 4hr CIT; 30 min WIT ${ }^{\#}$ & $\mathrm{CD} 47$ & [13] \\
\hline \multicolumn{5}{|c|}{ Targeting Innate Immunity, Necroptosis and Cell Stress Pathways } \\
\hline Farraar et al. & 2012 & Syngeneic; 30min CIT* $^{*}$ & TLR2 & [14] \\
\hline Lau et al. & 2013 & Allogeneic; 35-40min (WIT+CIT) & RIPK3 & [15] \\
\hline Rink et al. & 2018 & Syngeneic; 4hr CIT; 30 min WIT ${ }^{\#}$ & NO-HDL-NPs & [16] \\
\hline Sörensen et al. & 2011 & Allogeneic; $45 \mathrm{~min} \mathrm{CIT}^{*} \dagger$ & $\mathrm{B} \beta(15-42)$ & [17] \\
\hline
\end{tabular}

Note: \#Both native kidneys of recipient mice were removed during transplant surgery. *One native kidney of recipient mice was kept in situ to 4-5 days post-transplant. † Warm ischemic time was not reported. CIT: cold ischemic time; WIT: warm ischemic time; TLR: Toll-like receptor; RIPK: receptor-interacting protein kinase; NO-HDL-NPs: nitric oxide-delivering high-density lipoproteinlike nanoparticles.

\section{Hormonal Influence}

Sex disparities in kidney IRI tolerance have been proven in both animal systems and human studies $[9,18]$. By utilizing a mouse KT model with 8hr CIT, Aufhauser et al. [9] showed that female recipient mice had improved renal ischemia tolerance compared to male recipient mice, which correlated with better transplant outcomes. They found that renal IRI was exacerbated in female mice with estrogen receptor $\alpha$ deficiency, while female mice receiving supplemental estrogen prior to ischemia were protected [9]. Moreover, Gueler et al. [10] showed that the EA-230, an oligopeptide derived from $\beta$-human chorionic gonadotropin ( $\beta$-hCG) lysates, ameliorated renal ischemic injury, improved renal allograft function, and prolonged survival in a mouse model of KT with $1 \mathrm{hr}$ CIT. These studies suggest the potential benefits of hormone supplements in kidney transplant IRI [10].

\section{Complement System}

Several experimental models of acute kidney injury (AKI) induced by clamping have demonstrated a clear-cut role for complement system in renal IRI $[19,20]$ yet only a few studies have tested the effect of complement inhibitors in kidney transplant IRI models [12,21]. Using syngeneic mouse KT models with $4 \mathrm{hr}$ CIT, Zheng et al. showed that the administration of siRNA cocktail solution targeting complement 3 , RelB, and Fas significantly reduced the expression of proinflammatory cytokines, interleukin-6 (IL-6), and tumor necrosis factor- $\alpha$ (TNF- $\alpha$ ), decreased cell apoptosis, and improved renal function [11]. Recently, Casiraghi et al. [12] showed that BALB/c kidneys transplanted into complement factor b-deficient B6 recipients exhibited reduced IRI and diminished $\mathrm{T}$ cell-mediated rejection. The administration of anti-complement factor B antibody to recipients in early post-transplant phases ameliorated both IRI and early adaptive immune responses [12]. 


\section{Tubular Repair and Regeneration}

It has been accepted that proximal tubule regeneration postAKI occurs from intrinsic tubule cells [22]. Therefore, seeking strategies to promote tubular repair and regeneration holds promise for ameliorating renal graft damage. Our collaborative studies with Isenberg's Lab showed that the treatment of renal tubular epithelial cells with a CD47 blocking antibody or CD47targeting siRNA increased expression of self-renewal transcription factors and promoted cell proliferation [13]. Further studies in mouse models of KT with $4 \mathrm{hr}$ CIT indicated that the treatment with a CD47 blocking antibody increased self-renewal transcription factor expression, decreased tissue damage, and improved renal function compared to that in control mice [13].

\section{Targeting Innate Immunity, Necroptosis, and Cell Stress Pathways}

It is known that IRI triggers a vast array of inflammatory mediators that activate innate immune responses. Toll-like receptors (TLRs) are critical molecules involved in inflammation. By using a mouse KT models with $1 \mathrm{hr}$ CIT, Farrar et al. showed that inhibition of TLR2 with a therapeutic agent (OPN301) significantly decreased acute tubular necrosis and improved renal graft function compared to controls [14]. Necroptosis, a term for programmed cell necrosis, is an emerging entity that might be involved in transplant IRI. This process is characterized by a pathway dependent on the receptor-interacting protein kinase 1 (RIPK1)-RIPK3 complex [23]. A recent study by Lau et al. showed that BALB/c mice receiving RIPK3-/- kidneys had improved renal function and longer survival compared to controls, suggesting that inhibition of necroptosis in donor organs may provide a clinical benefit [15].

Other therapeutic targets testing in the mouse KT models with prolonged CIT included the nitric oxide-delivering highdensity lipoprotein-like nanoparticles (NO-HDL-NPs) and B $\beta(15-$ 42), a breakdown product of fibrin that could inhibit leukocyteendothelial adhesion, as summarized in Table 1 and [16, 17] Besides the therapeutic approaches summarized above, other potential targets that can be tested in the modified mouse models of KT include the mitochondrial dysfunction [24], endoplasmic reticulum stress [25], and lipid peroxidation [26]. With regard to the mouse model of KT IRI, the major limitation is that the surgical procedure is technically challenging and requires extensive microsurgical training. Few people in the world are able to perform this procedure, making it less convenient for translational research. Nevertheless, this model is very attractive as it is powerful and clinically-relevant for the mechanistic investigations and for testing new strategies, thereby offering a great opportunity for identifying new therapies for transplant IRI. Furthermore, compared to mouse AKI models in which no transplant procedure is included, mouse KT IRI model can efficiently test the mechanisms of kidney intrinsic factors (donor) versus extrinsic factors (recipient).

\section{Conclusion}

The progress of therapies in KT IRI relies on a better understanding the pathophysiology of renal injury and repair. Mouse models of KT combined with extended IRI could serve as a powerful tool for exploring the mechanisms of renal graft injury and for testing new therapies for transplant recipients.

\section{Acknowledgment}

The author would like to acknowledge the support from members of the Comprehensive Transplant Center Microsurgery Core at Northwestern University. This work is partially supported by NIH/NIAID P01AI112522 and R01AI112911. The author would like to thank Nate Esparza for proofreading the article.

\section{References}

1. Siedlecki A, Irish W, Brennan DC (2011) Delayed graft function in the kidney transplant. Am J Transplant 11(11): 2279-2296.

2. Salvadori M, Rosso G, Bertoni E (2015) Update on ischemia-reperfusion injury in kidney transplantation: Pathogenesis and treatment. World J Transplant 5(2): 52-67.

3. Mori DN, Kreisel D, Fullerton JN, Gilroy DW, Goldstein DR (2014) Inflammatory triggers of acute rejection of organ allografts. Immunol Rev 258(1): 132-144.

4. Zhao H, Alam A, Soo AP, George AJT, Ma D (2018) Ischemia-Reperfusion Injury Reduces Long Term Renal Graft Survival: Mechanism and Beyond. E Bio Medicine 28: 31-42.

5. Wu WK, Famure O, Li Y, Kim SJ (2015) Delayed graft function and the risk of acute rejection in the modern era of kidney transplantation. Kidney Int 88(4): 851-858.

6. Rege A, Irish B, Castleberry A, Vikraman D, Sanoff S, et al. (2016) Trends in Usage and Outcomes for Expanded Criteria Donor Kidney Transplantation in the United States Characterized by Kidney Donor Profile Index. Cureus 8(11): 887.

7. Dayoub JC, Cortese F, Anzic A, Grum T, De Magalhaes JP (2018) The effects of donor age on organ transplants: A review and implications for aging research. Exp Gerontol 110:230-240.

8. Incerti D, Summers N, Ton TGN, Boscoe A, Chandraker A, et al. (2018) The Lifetime Health Burden of Delayed Graft Function in Kidney Transplant Recipients in the United States. MDM Policy Pract 3(1).

9. Aufhauser DD, Wang Z, Murken DR, Bhatti TR, Wang Y, et al. (2016) Improved renal ischemia tolerance in females influences kidney transplantation outcomes. J Clin Invest 126(5): 1968-1977.

10. Gueler F, Shushakova N, Mengel M, Hueper K, Chen R, et al. (2015) A novel therapy to attenuate acute kidney injury and ischemic allograft damage after allogenic kidney transplantation in mice. PLos one 10(1) e0115709.

11. Zheng X, Zang G, Jiang J, He W, Johnston NJ, et al. (2016) Attenuating Ischemia-Reperfusion Injury in Kidney Transplantation by Perfusing Donor Organs With siRNA Cocktail Solution. Transplantation 100(4): 743-752.

12. Casiraghi F, Azzollini N, Todeschini M, Fiori S, Cavinato RA, et al. (2017) Complement Alternative Pathway Deficiency in Recipients Protects Kidney Allograft From Ischemia/Reperfusion Injury and Alloreactive T Cell Response. Am J Transplant 17(9): 2312-2325.

13. Rogers NM, Zhang ZJ, Wang JJ, Thomson AW, Isenberg JS (2016) CD47 regulates renal tubular epithelial cell self-renewal and proliferation following renal ischemia reperfusion. Kidney Int 90(2): 334-347. 
14. Farrar CA, Keogh B, Mccormack W, O Shaughnessy A, Parker A, et al. (2012) Inhibition of TLR2 promotes graft function in a murine model of renal transplant ischemia-reperfusion injury. FASEB J 26(2): 799-807.

15. Lau A, Wang S, Jiang J, Haig A, Pavlosky A, et al. (2013) RIPK3-mediated necroptosis promotes donor kidney inflammatory injury and reduces allograft survival. Am J Transplant 13(11): 2805-2818.

16. Rink JS, Sun W, Misener S, Wang JJ, Zhang ZJ, et al. (2018) Nitric OxideDelivering High-Density Lipoprotein-like Nanoparticles as a Biomimetic Nanotherapy for Vascular Diseases. ACS Appl Mater Interfaces 10(8): 6904-6916.

17. Sorensen I, Rong S, Susnik N, Gueler F, Shushakova N, et al. (2011) Bbeta(15-42) attenuates the effect of ischemia-reperfusion injury in renal transplantation. J Am Soc Nephrol 22(10):1887-1896.

18. Kim SJ, Gill JS (2009) H-Y incompatibility predicts short-term outcomes for kidney transplant recipients. J Am Soc Nephrol 20(9): 2025-2033.

19. Peng Q Li K, Smyth LA, Xing G, Wang N, et al. (2012) C3a and C5a promote renal ischemia-reperfusion injury. J Am Soc Nephrol 23(9): 1474-1485.

20. Kotimaa JP, Van Werkhoven MB, O Flynn J, Klar Mohamad N, Van Groningen J, et al. (2015) Functional assessment of mouse complement pathway activities and quantification of $\mathrm{C} 3 \mathrm{~b} / \mathrm{C} 3 \mathrm{c} / \mathrm{iC} 3 \mathrm{~b}$ in an experimental model

\section{ISSN: 2574-1241}

DOI: 10.26717/BJSTR.2019.14.002617

Zheng Jenny Zhang. Biomed J Sci \& Tech Res

(C)

Submission Link: https://biomedres.us/submit-manuscript.php of mouse renal ischaemia/reperfusion injury. J Immunol Methods 419: $25-34$.

21. Gueler F, Rong S, Gwinner W, Mengel M, Brocker V, et al. (2008) Complement 5 a receptor inhibition improves renal allograft survival. J Am Soc Nephrol 19(12): 2302-2312.

22. Berger K, Moeller MJ (2014) Mechanisms of epithelial repair and regeneration after acute kidney injury. Semin Nephrol 34(4): 394-403.

23. Moriwaki K, Chan FK (2014) Necrosis-dependent and independent signaling of the RIP kinases in inflammation. Cytokine Growth Factor Rev 25(2): 167-174

24. Emma F, Montini G, Parikh SM, Salviati L (2016) Mitochondrial dysfunction in inherited renal disease and acute kidney injury. Nat Rev Nephrol 12(5): 267-280.

25. Shu S, Zhu J, Liu Z, Tang C, Cai J, et al. (2018) Endoplasmic reticulum stress is activated in post-ischemic kidneys to promote chronic kidney disease. EBioMedicine 37: 269-280.

26. Palipoch S (2013) A review of oxidative stress in acute kidney injury: protective role of medicinal plants-derived antioxidants. Afr J Tradit Complement Altern Med 10(4): 88-93.

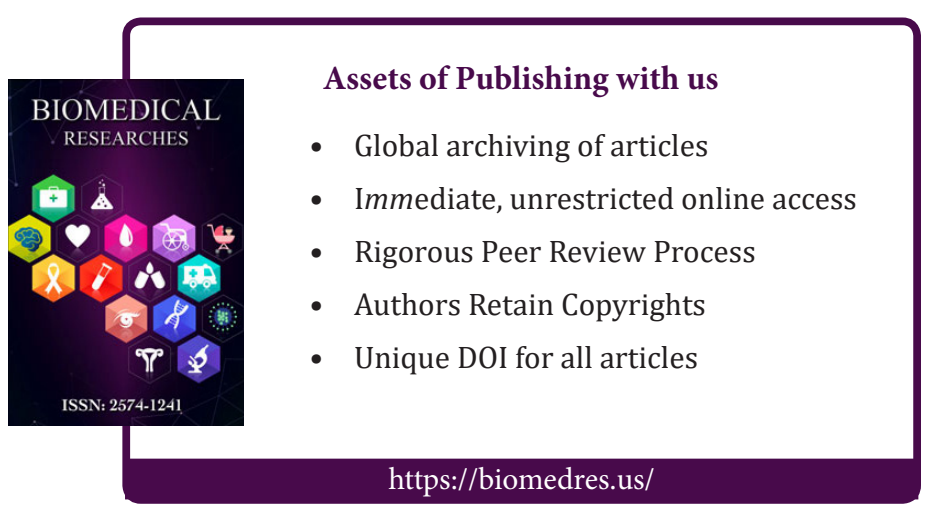

